\title{
Editorial: epigenetic regulation of plant development and stress responses
}

\author{
Tarek Hewezi ${ }^{1}$
}

Received: 31 October 2017 / Accepted: 5 November 2017 / Published online: 20 November 2017

c) Springer-Verlag GmbH Germany, part of Springer Nature 2017

Epigenetic components, including cytosine methylation, histone modifications, and small RNA accumulation are key marks regulating various plant traits including growth, development, and response to biotic and abiotic stimuli. This special issue, which includes two opinion papers, five reviews, and eight research articles, highlights the importance and functions of epigenetic mechanisms in various developmental and stress contexts.

Weinhold (2018) discusses the importance of epigenetics in the transgenerational adaptive response to environmental stimuli. Relevant ecologically motivated experimental systems, which include appropriate plant species and stress treatments, can illuminate the dynamics of epigenetic transgenerational stress adaption and how plants could benefit from their stress memory. DNA methylation and histone modifications are known to profoundly impact gene expression but their impacts on the 3-dimensional structure of the chromosomes are often overlooked in epigenetic studies. In their opinion paper, Dukowic-Schulze et al. (2017) highlight the role of chromatin modifications during the sexual reproduction of plants with special emphasis on the 3-dimensional chromosome reorganization mediated by these epigenetic modifications in actively dividing cells.

Epigenetic modifications are reversible and endow rapid response mechanisms to allow plants to combat pathogen and environmental stress. In this issue, Hewezi et al. (2017) provide comprehensive analysis of plant methylome changes induced by various phytopathogens that underscores the importance of DNA demethylation as a common feature of plant response during the susceptible interactions. The role of active DNA demethylation in various aspects of plant

Communicated by Günther Hahne.

Tarek Hewezi

thewezi@utk.edu

1 Department of Plant Sciences, University of Tennessee, Knoxville, TN 37996, USA development including pollen tube formation, embryogenesis, fruit ripening, stomatal development, and nodule formation, is surveyed by Li et al. (2017). The importance of epigenetic regulation of other agriculturally important traits such as vernalization, hybrid vigor, and self-incompatibility is also illustrated by Itabashi et al. (2017). Rice, as a model for cereal epigenomic studies, appears to have enormous plasticity for epigenomic alteration. Banerjee and Roychoudhury (2017) thoroughly discuss the association among various components of the rice epigenome and plant growth, flowering, and reproduction, as well as responses to stress. Recent research shows that various classes of small RNAs are key epigenetic regulators. Kumar et al. (2017) review the current state of knowledge on the role of small RNAs in plant stress tolerance.

Despite the nature of the biochemical signals that induce changes in plant epigenome under a wide range of developmental and stress contexts, several unknowns remain. Nonetheless, there appears to be a tight association between epigenetic modifications and phytohormone signaling. In this special issue, Wakeel et al. (2017) reviewed the potential involvement of histone acetylation/deacetylation in regulating auxin biosynthesis and signaling. In this context, Zheng et al. (2017) report a role of gibberellins in increasing the level of histone acetylation, thereby decondensing $45 \mathrm{~S}$ rDNA chromatin in aleurone layers during maize seed germination. Another study by Guo et al. (2017) reports on a novel function of a tomato histone deacetylase gene in the regulation of fruit ripening via inhibiting ethylene biosynthesis and carotenoid accumulation.

Various components of epigenetic mechanisms are known to function in a synchronized manner to regulate gene expression. López-Galiano et al. (2017) provide interesting insight into a possible coordinated function of a miRNA gene and histone modifications in regulating the expression of a WRKY transcription factor in response to various biotic and abiotic stresses. The association between various histone epigenetic marks and transcriptional regulation 
of defense-related genes during the early plant response to infection by the necrotrophic fungus Botrytis cinerea is reported by Crespo-Salvador et al. (2017).

Silencing the expression of stably integrated transgenes at the transcription or post-transcriptional levels is a very common phenomenon in plants. Transcriptional silencing of transgenes is generally associated with hypermethylation in the transgene promoter region, whereas post-transcriptional silencing involves degradation of transgene transcripts. Tyč et al. (2017), which was published in August issue 2017 examine the effect of an inhibitor of DNA methylation on the reactivation of two transcriptionally silenced reporter genes. Interestingly, they found that re-silencing of the transgene can be spontaneously triggered via in vitro de novo regeneration. In this perspective, Coronel et al. (2017) also provide new evidence that tissue culture can induce high levels of epigenetic variation.

RNA interference (RNAi) is also considered as an epigenetic mechanism that leads to the generation of small RNAs, which mediate gene silencing at post-transcriptional level in a sequence-specific manner. RNAi technology has tremendous potential for plant disease resistance. In this special issue, Yang et al. (2017) describe the production of genetically engineered soybean lines with enhanced and stable resistance to various Potyvirus strains by introducing an inverted repeat corresponding to the soybean mosaic virus P3 cistron.

This special issue includes some of the most exciting results in plant biology, which happen to also have some of the most promising impacts on agriculture. During the past few years, we have learned that a new genetic world exists beyond Mendelian genetics. During this great expansive phase of plant epigenetic research, we can expect to learn about the biological significance of various epigenetic marks, which will certainly lead to more sustainable agriculture.

Acknowledgements I would like to thank the authors, reviewers, and journal editorial staff who have contributed to this special issue.

\section{References}

Banerjee A, Roychoudhury A (2017) The gymnastics of epigenomics in rice. Plant Cell Rep. https://dx.doi.org/10.1007/ s00299-017-2192-2

Coronel CJ, González AI, Ruiz ML, Polanco C (2017) Analysis of somaclonal variation in transgenic and regenerated plants of
Arabidopsis thaliana using methylation related metAFLP and TMD markers. Plant Cell Rep. https://dx.doi.org/10.1007/ s00299-017-2217-x

Crespo-Salvador O, Escamilla-Aguilar M, López-Cruz J, Gerardo López-Rodas G, González-Bosch C (2017) Determination of histone epigenetic marks in Arabidopsis and Tomato genes in the early response to Botrytis cinerea. Plant Cell Rep. https://dx.doi. org/10.1007/s00299-017-2218-9

Dukowic-Schulze S, Liu C, Chen C (2017) Not just gene expression: 3D implications of chromatin modifications during sexual plant reproduction. Plant Cell Rep. https://dx.doi.org/10.1007/ s00299-017-2222-0

Guo JE, Hu Z, Yu X, Li A, Li F, Wang Y, Tian S, Chen G (2017) A histone deacetylase gene, SIHDA3, acts as a negative regulator of fruit ripening and carotenoid accumulation. Plant Cell Rep. https://dx.doi.org/10.1007/s00299-017-2211-3

Hewezi T, Pantalone V, Bennett M, Stewart CN, Burch-Smith TM (2017) Phytopathogen-induced changes to plant methylomes. Plant Cell Rep. https://dx.doi.org/10.1007/s00299-017-2188-y

Itabashi E, Osabe K, Fujimoto R, Kakizaki T (2017) Epigenetic regulation of agronomical traits in Brassicaceae. Plant Cell Rep. https:// dx.doi.org/10.1007/s00299-017-2223-z

Kumar V, Khare T, Shriram V, Wani SH (2017) Plant small RNAs: the essential epigenetic regulators of gene expression for saltstress responses and tolerance. Plant Cell Rep. https://dx.doi. org/10.1007/s00299-017-2210-4

Li Y, Kumar S, Qian W (2017) Active DNA demethylation: mechanism and role in plant development. Plant Cell Rep. https://dx.doi. org/10.1007/s00299-017-2215-z

López-Galiano MJ, González-Hernández AI, Crespo-Salvador O, Rausell C, Real MD, Escamilla M, Camañes G, García-Agustín P, González-Bosch C, García-Robles I (2017) Epigenetic regulation of the expression of WRKY75 transcription factor in response to biotic and abiotic stresses in Solanaceae plants. Plant Cell Rep. https://dx.doi.org/10.1007/s00299-017-2219-8

Tyč D, Nocarová E, Sikorová L, Fischer L (2017) 5-Azacytidine mediated reactivation of silenced transgenes in potato (Solanum tuberosum) at the whole plant level. Plant Cell Rep. https://dx.doi. org/10.1007/s00299-017-2155-7

Wakeel A, Ali I, Khan AR, Wu M, Upreti S, Liu D, Liu B, Gan Y (2017) Involvement of histone acetylation and deacetylation in regulating auxin responses and associated phenotypic changes in plants. Plant Cell Rep. https://dx.doi.org/10.1007/ s00299-017-2205-1

Weinhold A (2018) Transgenerational stress adaption-an opportunity for ecological epigenetics. Plant Cell Rep. https://dx.doi. org/10.1007/s00299-017-2216-y

Yang X, Niu L, Zhang W, Yang J, Xing G, He H, Guo D, Du Q, Qian X, Yao Y, Li Q (2017) RNAi-mediated SMV P3 cistron silencing confers significantly enhanced resistance to multiple Potyvirus strains and isolates in transgenic soybean. Plant Cell Rep. https:// dx.doi.org/10.1007/s00299-017-2186-0

Zheng X, Hou H, Zhang H, Yue M, Hu Y, Li L (2017) Histone acetylation is involved in GA-mediated 45S rDNA decondensation in maize aleurone layers. Plant Cell Rep. https://dx.doi.org/10.1007/ s00299-017-2207-z 